\title{
Family-based exome sequencing and case- control analysis implicate CEP41 as an ASD gene
}

Ashok Patowary 10', So Yeon Won², Shin Ji Oh², Ryan R Nesbitt', Marilyn Archer', Debbie Nickerson³, Wendy H. Raskind ${ }^{1,4}$, Raphael Bernier ${ }^{1}$, Ji Eun Lee ${ }^{2,5}$ and Zoran Brkanac ${ }^{1}$

\begin{abstract}
Autism Spectrum Disorder (ASD) is a complex neurodevelopmental disorder with a strong genetic component. Although next-generation sequencing (NGS) technologies have been successfully applied to gene identification in de novo ASD, the genetic architecture of familial ASD remains largely unexplored. Our approach, which leverages the high specificity and sensitivity of NGS technology, has focused on rare variants in familial autism. We used NGS exome sequencing in 26 families with distantly related affected individuals to identify genes with private gene disrupting and missense variants of interest (VOI). We found that the genes carrying VOls were enriched for biological processes related to cell projection organization and neuron development, which is consistent with the neurodevelopmental hypothesis of ASD. For a subset of genes carrying VOls, we then used targeted NGS sequencing and gene-based variant burden case-control analysis to test for association with ASD. Missense variants in one gene, CEP41, associated significantly with ASD $\left(p=6.185^{\mathrm{e}-05}\right)$. Homozygous gene-disrupting variants in CEP41 were initially found to be responsible for recessive Joubert syndrome. Using a zebrafish model, we evaluated the mechanism by which the CEP41 variants might contribute to ASD. We found that CEP41 missense variants affect development of the axonal tract, cranial neural crest migration and social behavior phenotype. Our work demonstrates the involvement of CEP41 heterozygous missense variants in ASD and that biological processes involved in cell projection organization and neuron development are enriched in ASD families we have studied.
\end{abstract}

\section{Introduction}

Autism Spectrum Disorders (ASD) comprise a heterogeneous group of complex neurodevelopmental conditions diagnosed based on the presence of deficits in social communication and interactions along with restricted, repetitive behaviors, interests or activities (DSM5) ${ }^{1}$. Family and twin studies have provided strong evidence for inherited genetic factors in the etiology of $\mathrm{ASD}^{2,3}$. Despite

\footnotetext{
Correspondence: Ji Eun Lee (jieun.lee@skku.edu) or

Zoran Brkanac (zbrkanac@uw.edu)

${ }^{1}$ Department of Psychiatry and Behavioral Sciences, University of Washington, Seattle, WA, USA

${ }^{2}$ Department of Health Sciences and Technology, SAIHST, Sungkyunkwan

University, Seoul, Korea

Full list of author information is available at the end of the article.

These authors contributed equally: Ashok Patowary, So Yeon Won.
}

high heritability, the genetic architecture of ASD remains elusive. Advances in array and later sequencing technologies accelerated elucidation of the contribution of de novo copy number variations ( $\mathrm{CNVs}$ ) and de novo singlenucleotide mutations to the molecular basis of sporadic ASD and have estimated that there are hundreds to thousands of ASD risk $\operatorname{loci}^{4-9}$. Genomic studies of sporadic autism have also paved the way for using systems biology approaches, such as pathway enrichment and gene co-expression profiling, to implicate neuronal signaling, synaptic transmission, chromatin biology, transcription regulation, cellular proliferation, projection and motility and GTPase/Ras signaling in the etiology of $\mathrm{ASD}^{10-13}$.

\section{(c) The Author(s) 2018}

(c) (i) Open Access This article is licensed under a Creative Commons Attribution 4.0 International License, which permits use, sharing, adaptation, distribution and reproduction in any medium or format, as long as you give appropriate credit to the original author(s) and the source, provide a link to the Creative Commons license, and indicate if changes were made. The images or other third party material in this article are included in the article's Creative Commons license, unless indicated otherwise in a credit line to the material. If material is not included in the article's Creative Commons license and your intended use is not permitted by statutory regulation or exceeds the permitted use, you will need to obtain permission directly from the copyright holder. To view a copy of this license, visit http://creativecommons.org/licenses/by/4.0/. 
The frequent detection of de novo variants in ASD supports the role for rare variants in ASD but, in contrast to the studies of sporadic autism, in familial ASD gene discovery has not been as productive ${ }^{14}$. Progress has been made in identification of genes with recessive inheritance in ASD due to power of homozygosity mapping in consanguineous families ${ }^{15-17}$. Studies of individual ASD families, where pedigrees were compatible with Mendelian dominant inheritance, have demonstrated the suitability of exome sequencing to identify candidate ASD variant ${ }^{18-20}$. The medium scale studies that explored the role of dominant inheritance in between 7 and 40 families have identified large numbers of candidate genes and consequently have not followed up with case-control analyses to determine the significance of such findings ${ }^{21-23}$. The two large studies used whole-genome sequencing (WGS) in 85 and 878 families for ASD gene identification ${ }^{9,24}$. The WGS studies utilized numerous analytical approaches including non-coding DNA, exome, CNV, single-nucleotide variant, de-novo, recessive and dominant inheritance analysis. These studies confirmed substantial genetic heterogeneity in familial autism. They ultimately lacked statistical power to identify novel ASD genes as it is estimated that sample sizes to obtain statistical power in exome studies are similar to genome-wide association studies (GWAS) and will probably require more than 25,000 cases $^{25}$. We have previously shown that the study design which utilizes ASD families with distantly related cases (i.e. second and third cousin families) allows for identification of a small number of candidate genes that can be evaluated in case control study to identify association with ASD $^{26}$.

In our current study, we focused on 26 families with at least one affected cousin pair to identify private gene disrupting and missense variants of interest (VOI) in each family. The genes we identified are enriched for "cell projection organization" and "neuron development", which are categories we found enriched in known ASD genes as well. Furthermore, for a subset of candidate genes we performed a gene-based case-control analysis in a larger sample and found significant association with CEP41, which we further confirmed using the familybased transmission disequilibrium test (TDT). We then used a zebrafish model to characterize the functional effects of the CEP41 variants identified in the case-control study. The zebrafish studies showed that the expression of the variants in embryos induces axonal defects and also affects cranial neural crest (CNC) cell migration. Furthermore, zebrafish carrying the CEP41 variants we identified in ASD cases exhibited deficits in social behavior.

In summary, our familial exome sequencing analysis identified novel ASD candidate genes that are enriched for neuronal development and cell projection organization. Case-control analyses of a subset of candidate genes revealed a significant ASD association with CEP41, which was confirmed by TDT. Furthermore, we showed in a zebrafish model that ASD-associated CEP41 variants affect axonal development, $\mathrm{CNC}$ migration and social behavior.

\section{Methods \\ Study sample}

For the family-based exome sequencing study, we identified 26 ASD families with affected first cousins from the NIMH repository (https://www.nimhgenetics.org/). For each family, we obtained DNA from the affected cousins and their affected siblings when available, for a total of 61 affected subjects. The pedigrees for the families used in the study are shown in Supplementary Information.

For case control studies, we obtained 1143 familial Caucasian cases and 1168 Caucasian controls as described previously ${ }^{26}$. In brief, the subjects were obtained from the NIMH repository, a University of Washington (UW) multiplex autism collection and a UW study on dyslexia. For both NIMH and UW ASD subjects, the diagnostic status was determined by gold-standard ADOS and ADI$\mathrm{R}$ assessment followed by expert clinical judgment using all available information. The NIMH control sample consists of adults who completed an online short selfreport assessment to exclude severe psychiatric disorders, but were not specifically screened for autism. The UW controls were ascertained for dyslexia by comprehensive testing, and screened for absence of ASD with a questionnaire. All samples from the NIMH collection and UW were utilized in concordance with Institutional Review Board approvals from all participating institutions for sample and data sharing.

\section{Whole-exome sequencing, variant calling, annotation and prioritization}

Nimblegen SeqCap EZ Human Exome Library v2.0 (Roche, Basel, Switzerland) was used to capture the exomes, followed by paired-end $50 \mathrm{bp}$ sequencing on an Illumina HiSeq2000 sequencing platform. Sequences were generated in the UW Genome Sciences Center for Mendelian Genomics ${ }^{27}$. Raw sequencing files were processed for quality control with FastQC (http://www. bioinformatics.babraham.ac.uk/projects/fastqc/). Exomecaptured sequencing reads were aligned to NCBI human reference genome GRCh37 (hg19) using Burrows-Wheeler Aligner (BWA v0.7.10) ${ }^{28}$. Aligned reads were subsequently processed using Picard v1.118 (http://picard.sourceforge.net) for duplicate removal. Thereafter, Genome Analysis Toolkit (GATK v3.2) was used for variant calling ${ }^{29}$. ANNOVAR ${ }^{30}$ (v2014) was used for annotating the single-nucleotide variants called by GATK. 
To identify VOIs we used multistep filtering. Initial filtering included the removal of intergenic and $3^{\prime} / 5^{\prime}$ UTR variants, non-splice related intronic variants and synonymous variants. Variants were further filtered based on 1000 Genomes (1KG) "European", Exome Sequencing Project (ESP) European American and dbSNP databases. For the most stringent frequency filtering, we retained only "private" variants, which we define as variants not present in any of the data sets (dbSNP132, 1KG (2012 April) and ESP). Finally, to be marked as a VOI, the variant needed to be present in all affected individuals in a family. Predictions on variant deleteriousness based on SIFT, Poly-Phen-2 and GERP scores were obtained through ANNOVAR (v2014). Taking into consideration bioinformatics predictions of variant deleteriousness, to select genes for case-control association studies, we performed manual PubMed searches and evaluated genes based on available information on brain expression and function.

Targeted gene sequencing, variant calling and annotation We employed molecular inversion probes (MIPs) for targeted capture of the coding region of the selected genes $^{31}$. We designed MIPs for protein-coding regions of 13 genes and performed sequencing and analysis as described previously (details in Supplementary Information). Sequence reads were aligned to NCBI human reference genome GRCh37 (hg19) using Burrows-Wheeler Aligner $\left(\right.$ BWA v0.7.10) ${ }^{28}$. Variants were called with $\mathrm{GATK}^{29}$ (v3.2) unified genotyper. Thereafter, ANNOVAR ${ }^{30}$ (v2015) was used to annotate the single-nucleotide variants as nonsynonymous, splice, stop gain, stop lost or synonymous variants. For MIP sequencing analysis, we used slightly less stringent criteria to identify "rare" variants, which we defined as variants with frequency $<0.01$ in $1 \mathrm{KG}$ European samples. For the case-control association analysis, we used rare protein altering variants (nonsynonymous, splice, stop gain, stop lost).

\section{Variant validation and evaluation in family members}

Variants in the genes selected for case-control analysis and rare CEP41 variants identified in cases and controls were confirmed by Sanger sequencing as previously described $^{23}$. For cases with CEP41 variants, available affected siblings were evaluated for presence of the variant allele using Sanger sequencing.

\section{Association analysis}

For the case-control study, we performed gene-based variant burden association analysis ${ }^{32}$. For each gene, we aggregated all rare protein-altering variants. Significance of the burden association was determined with one-sided Fisher exact test.

For CEP41, we performed TDT analyses ${ }^{33}$ to further evaluate the association with ASD. TDT uses $X^{2}$ goodness of fit statistics to determine if there is a preferential transmission of a risk allele to the affected cases. In the TDT analysis, we used affected siblings of cases with rare protein-altering variants in CEP41, under the assumption that if CEP41 variants contribute to the phenotype, we should see over transmission of risk variants to affected siblings. This analysis incorporated a rare-variant extension of the TDT. We used a TDT-burden of rare variants that counts the number of minor-allele-transmission events to affected siblings.

\section{Functional enrichment analysis}

Genes with VOIs identified in exome sequencing were subjected to WebGestalt ${ }^{34}$ for functional enrichment analysis using the Overrepresentation Enrichment Analysis (ORA). We interrogated the Gene Ontology (GO) database for enrichment in three categories: "Biological Process", "Cellular Component" and "Molecular Function". The focus of enrichment analysis was to identify broad biological processes that are involved in ASD. As the current estimates for the number of genes in ASD ranges from hundreds to thousands ${ }^{4-9}$, to reflect ASD number of genes estimates, we set the parameters used in the analysis to Minimum Number of Genes $=500$ and Maximum Number of Genes $=1500$. False Discovery Rate (FDR) analysis was performed by Benjamini-Hochberg procedure as a measure of control for false positives. Enrichment was considered significant for FDR below 0.05 .

For enrichment analysis of known ASD genes, we used the SFARI gene ${ }^{35}$ database (https://gene.sfari.org/). In our analysis, we included SFARI genes for syndromic disorders that have ASD as part of the phenotype (Category S) and genes that are identified as having the strongest evidence for involvement based on rigorous statistical case-control comparisons (Categories 1 and 2). For SFARI ASD gene enrichment analysis, we used the same analysis parameters as for VOI gene analysis.

\section{DNA plasmid construction}

Human wild-type (WT) CEP41, purchased from Dharmacon, was mutated to generate variants found in the case-control study using QuickChange II Site-Directed Mutagenesis Kit (Agilent, \#200524, USA). Zebrafish WT cep41 was amplified with cDNAs obtained from 3 days post-fertilization ( $\mathrm{dpf}$ ) zebrafish and mutated to generate MO-insensitive construct. The human and zebrafish CEP41 plasmid constructs were subcloned into the $\mathrm{pCS} 2+$ vector for expression in zebrafish.

\section{Zebrafish housing and manipulations}

Adult WT zebrafish (AB strain) were maintained with a cycle of $13 \mathrm{~h}$ light and $11 \mathrm{~h}$ dark in the automatic system (Genomic-Design, Korea) at $28.5^{\circ} \mathrm{C}$ and pH 7.0-7.9. The 
zebrafish embryos were collected by natural breeding and incubated in clean Petri dishes containing E3 medium (297.7 mM NaCl, $10.7 \mathrm{mM} \mathrm{KCl}, 26.1 \mathrm{mM} \mathrm{CaCl} 2$ and $24.1 \mathrm{mM} \mathrm{MgCl}_{2}$ ) containing 1\% methylene blue (Samchun chemicals, M2662, Korea) at $28.5^{\circ} \mathrm{C}$. To inhibit the formation of melanin, which interferes with immunostaining, the zebrafish larvae were raised in E3 medium containing $0.2 \mathrm{mM} \mathrm{N}$-phenylthiourea (PTU; SigmaAldrich, p7629, USA).

\section{Microinjection into zebrafish}

To block the expression of zebrafish cep41, translationblocking antisense morpholino oligonucleotides (MOs) were designed and synthesized by GeneTools (Philomath, OR, USA). The MOs (5'-CATCTTCCAGCAGCAGAGC TTCGGC-3') were dissolved in nuclease-free water $(2.5 \mu \mathrm{g} / \mu \mathrm{l})$ and microinjected into zebrafish embryos using a gas-used microinjection system (World Precision Instruments, PV83 Pneumatic PicoPump, SYS-PV830, FL, USA). The efficiency of the designed MOs was verified by Western blot assay for CEP41 using control zebrafish and cep41 morphants. Capped mRNAs of WT or mutated human CEP41 variants were synthesized by mMESSAGE mMACHINE kit (Ambion, AM1340, USA). The in vitro synthesized mRNAs $(150-300 \mathrm{ng} / \mu \mathrm{l})$ were injected into zebrafish embryos together with cep41 MOs.

\section{Immunohistochemistry (IHC) and imaging of zebrafish embryos}

Zebrafish embryos at 6-13 somites stage were fixed in $4 \%$ paraformaldehyde (PFA) for $14 \mathrm{~h}$ at $4{ }^{\circ} \mathrm{C}$. After two times washing with $1 \times$ phosphate buffered saline (PBS), embryos were blocked with blocking solution (10\% normal goat serum (NGS) in $1 \times$ PBST $(0.5 \%$ Triton $\mathrm{X}$ 100 in $1 \times \mathrm{PBS})$ ) for $2 \mathrm{~h}$ at room temperature. Thirty-five hpf zebrafish embryos were dechorinated and soaked in $90 \%$ methanol for $1 \mathrm{~min}$ at $-20^{\circ} \mathrm{C}$. After washing with $1 \times$ PBS, the embryos were fixed in $4 \%$ PFA at $4{ }^{\circ} \mathrm{C}$ overnight and incubated in equal volume of $2 \times$ fixation buffer (0.2 M PBS containing $8 \%$ sucrose and $0.3 \mathrm{mM}$ $\mathrm{CaCl}_{2}$ ) for $2 \mathrm{~h}$ at $4{ }^{\circ} \mathrm{C}$. The embryos were rinsed with PBS after fixing and their eyes were removed before permeabilization with cold acetone $\left(-20^{\circ} \mathrm{C}\right.$ for $\left.20 \mathrm{~min}\right)$ and $10 \mu \mathrm{g} / \mathrm{ml}$ of proteinase $\mathrm{K}$ (RT for $10 \mathrm{~min}$ ). They were rinsed again with $0.1 \mathrm{M}$ PBS three times (for $5 \mathrm{~min}$ each). To prevent nonspecific binding of the secondary antibodies, embryos were kept for $1 \mathrm{~h}$ at $37^{\circ} \mathrm{C}$ in blocking solution ( $1 \times$ PBS, $1 \%$ BSA, $1 \%$ DMSO and $2 \%$ donkey serum). The zebrafish embryos were incubated with mouse anti-acetylated-tubulin antibodies (Sigma, T7451, MO, USA, 1:400) in blocking buffer ( $1 \times$ PBS, $1 \%$ BSA, $1 \%$ DMSO and $2 \%$ donkey serum) or with mouse anti-SOX10 antibodies (Santa Cruz, sc-365692, USA, 1:50) in blocking buffer ( $1 \times$ PBST and $5 \%$ NGS) at $4{ }^{\circ} \mathrm{C}$ overnight. These zebrafish embryos were then incubated with AlexaFlour ${ }^{\circledR} 488$-conjugated secondary antibodies (Invitrogen, mouse A11001, 1:500) at RT for $2 \mathrm{~h}$ after washing with PBST. The immunostained zebrafish embryos were mounted with $75 \%$ glycerol for photography using a fluorescence microscope (Nikon, SM21270, Japan) or embedded in 1\% low melting agarose (Mentos, M2070, Germany) dissolved in E3 medium for photography using a confocal microscope (Carl Zeiss, LSM700, Germany). The images were analyzed by NIS-Elements software (Nikon, Japan) or Zeiss ZEN imaging software (Carl Zeiss, Germany). For statistical analyses, zebrafish were allocated to experimental groups without randomization and blinding

\section{Zebrafish behavioral analysis}

Based on the established rodent models ${ }^{36}$ and proposed zebrafish models of $\mathrm{ASD}^{37}$, we developed a group preference test to evaluate zebrafish social behavior. In a group preference test, we evaluate if the target fish has a preference to spend more time close to a group, thereby indicating a preference for social interactions. We performed this paradigm in adult 4-month-old WT and cep $41+/-$ fish and in 5-6 dpf larvae injected with control $\mathrm{MO}$, cep41 MO and human CEP41 mRNAs variants.

Four-month-old adult zebrafish (WT and cep41+/-) were individually placed into a tank (length $18 \times$ height $7 \times$ width $8 \mathrm{~cm}$ ) filled with $500 \mathrm{ml}$ of system water. The tank was divided into two sections by placing a transparent plastic plate at the center. A group of six adults (three male and three female) was placed as a social cue in the first section and a target fish was placed in the second section. For measurement, the second section was divided into four zones based on proximity to the group. The target fish location was monitored for 17 min during light-on interval. Zebrafish larvae were individually placed into chambers $(7.3 \times 1 \times 3 \mathrm{~cm})$ filled with $10 \mathrm{ml}$ of E3 media, which were divided into two sections by placing a transparent glass plate at a quarter of length. A group of five larvae were offered as a social cue in the smaller section and the target larva was placed in the larger section, which was again divided into four zones for measurement purposes. The larva's zone preference during light-on conditions was monitored for $1 \mathrm{~h}$.

All recordings were performed using a Daniovision (Noldus, Wageningen, Netherlands) imaging system. The recorded video images were analyzed to quantify the cumulative time at each zone and measure target fish velocity using Ethovision XT software (Noldus, Wageningen, Netherlands). Five different target zebrafish larvae or six different target zebrafish adults were individually recorded in each analysis that was replicated. For statistical analyses, zebrafish were allocated to experimental groups without randomization and blinding. 


\section{RESULTS}

\section{Exome sequencing and analysis}

For 61 sequenced exomes, the average read depth was $64 \times$, with $82 \%$ of exome sequence covered at a depth greater than $20 \times$ and $93 \%$ covered at $8 \times$. Among all 61 samples, 98,430 coding single-nucleotide variants (SNVs) were identified of which 43,850 were nonsynonymous. On average, each exome contained approximately 23,500 SNVs, of which $\sim 12,500$ were nonsynonymous. The coverage statistics for exome sequencing is presented in Supplementary Table 1. Following variant function and frequency filtering, as described in Methods, we identified 0-17 VOIs per family, for a total of 145 VOIs in 139 genes (Supplementary Table 2). For VOIs, $137 / 145$ were present in a single family and four were present in two families. To facilitate the comparison of identified variants with other exome sequencing studies, we evaluated the predicted pathogenicity of the variants with SIFT, Polyhen2, GERP and CADD. Based on literature review which included information on gene expression in brain, gene function, involvement in neurogenetic disorders, and taking into consideration predicted variant pathogenicity scores, we selected 13 genes for the next-stage association study in a larger set of case and control samples: BAI2, CEP250, CEP41, CEP78, DCDC2, NFATC1, NTAN1, SCN10A, SH3BP4, SNPH, SPATA3, TMEM82 and ZNF638. Each of the 13 selected candidate genes was present in a single family only. Sanger sequencing confirmed the VOIs in all 13 candidate genes. All exome sequences reported in this study were deposited in NDAR (https://ndar.nih.gov/edit_collection. html?id=1919). Available phenotype information for the exome-sequenced samples is reported in Supplementary Table 3.

\section{Functional enrichment analysis}

Our hypothesis was that the shared VOIs identified in our exome-sequencing analysis are enriched for the genes that are relevant for brain development, as such genes are more likely to be involved in ASD susceptibility. Biological process analysis identified significant association for the involvement of genes containing VOIs with seven biological processes. These processes fall under two broad categories: "cell projection organization" (GO:0030030, $\mathrm{FDR}=0.0039)$ and "neuron development" (GO:0048666, $\mathrm{FDR}=0.0321$ ). The cell projection organization includes processes that result in assembly, arrangement or disassembly of cellular parts such as cilia or axon. The neuronal development GO process includes proteins involved in progression of a neuron in early development from initial commitment of the cell to neuronal fate to fully functional neuron. This includes the anatomical structures of neuron projection such as axons or dendrites. The enrichment analysis for cellular components identified association with seven GO terms including "cell junction" (GO:0030054, FDR $=0.0063)$, "microtubule cytoskeleton" (GO:0015630, FDR $=0.0098)$ and "cell surface" (GO:0009986, FDR $=0.0324)$. The cell junction is a specialized region where anchoring proteins extend through plasma membrane to form connections with neighboring cells or with extracellular matrix, while the microtubules (MTs) and associated proteins form the internal cell scaffolding. Molecular functions identified as enriched in our analysis include proteins involved in "kinase binding" (GO:0019900, FDR =0.0016). The GO is hierarchically organized and individual genes are included in multiple categories. The enriched genes and individual categories of biological processes, cellular components and molecular functions we identified are consequently overlapping as well. Thus, in Table 1, we presented a subset of enriched categories that contain all the genes with VOIs that were enriched. All categories enriched with FDR $<0.05$ are presented in Supplementary Table 4.

The enrichment analysis of 111 SFARI category S, 1 and 2 confirmed that enrichment categories we identified in VOI analysis are enriched in ASD as well. The majority of the categories overrepresented in VOI analysis, including "cell projection organization" and "neuron development", are also enriched in SFARI genes. All the enriched categories for SFARI genes are presented in Supplementary Table 5 .

\section{Targeted sequencing and variant burden association analysis}

To evaluate the statistical evidence for association of 13 selected candidate genes with ASD, we performed gene-based variant burden association analysis. Using MIP enrichment, the coding regions of these 13 genes were sequenced in 1143 cases and 1168 controls. After removal of duplicated and poorly captured samples, 1004 unrelated familial cases $(\mathrm{NIMH}$ cases $=800, \mathrm{UW}$ cases $=$ 204) and 1127 controls (NIMH controls $=924$, UW controls $=203$ ) were used for burden analysis. MIP sequencing and subsequent variant calling identified 992 variants within the 13 candidate genes. Following the multi-step approach described in the methods that includes variant annotation and frequency filtering, 277 rare protein-altering variants were included in casecontrol analyses (Supplementary Table 6).

The gene-based variant burden analysis identified a statistically significant association of CEP41 with ASD (Supplementary Table 7). We observed 18 CEP41 rare proteinaltering variants in cases and two in control samples $(p=$ $6.185 \mathrm{e}-05, \mathrm{OR}=10.26,95 \% \mathrm{CI}[2.37-44.36])$. One variant, p.P206A, was observed in 12 cases and one control ( $p=$ $8.527 \mathrm{e}-05, \mathrm{OR}=13.69,95 \% \mathrm{CI}[1.77-105.48])$. In addition, this p.P206A variant is present in the ExAC database at a frequency of 0.003719 in the European population $(p=$ 
0.0522, $\mathrm{OR}=1.62,95 \% \mathrm{CI}[0.91-2.91])$. All the CEP41 variants identified with MIP-based sequencing were confirmed by Sanger sequencing. The CEP41 variants are reported in Table 2. It is notable that rare variants identified in cases have bioinformatics scores as determined by SIFT, Polyphen, GERP and CADD that are more consistent with deleterious effects, as compared to a single variant p.A107G that was identified in controls.

The TDT was performed on 37 affected siblings of cases that had rare CEP41 variants. We found overtransmission of rare alleles to affected siblings: 27 rare alleles, 10 WT alleles $\left(\chi^{2}=6.9 ; p=0.008\right.$, Yates' corrected).

\section{ASD subject phenotype analysis}

Available phenotypic information for 54 subjects from 26 exome-sequenced families as well as their pedigrees is provided in Supplementary Information and Supplementary Table 3. For 28 subjects, we had verbal IQ, and for 30 subjects, we had non-verbal IQ information. In this sample, the mean verbal IQ was $85.5(\mathrm{SD}=26.22)$ and mean non-verbal IQ was 101.1 ( $\mathrm{SD}=17.11)$. Phenotype analysis for subjects with CEP41 mutations did not reveal any specific phenotype characteristics for available measures. Phenotype information for subjects with CEP41 mutations is shown in Supplementary Table 8.

CEP41 variants affect zebrafish axonal tract development

On the basis of prior work ${ }^{38}$ showing functional involvement of CEP41 in MTs that are important for axonal development, we wanted to evaluate the effect on axonal development of CEP41 variants found in ASD. We analyzed the brain axonal tracts at $35 \mathrm{~h}$ post-fertilization (hpf) zebrafish after exogenous expression of the variants by immunostaining with acetylated $\alpha$-tubulin antibodies. The axonal phenotypes in zebrafish injected with mRNA of human CEP41 variants were compared to those of zebrafish injected with cep41 MOs. The knockdown efficiency of the cep 41 MOs was validated by western blotting (Supplementary Figure 1a). Common morphological defects, such as curved tails and heart edema, of the cep41 morphants and the p.P206A variant-expressing zebrafish were in accordance with previously published results ${ }^{38}$ (Supplementary Figure 1b). The control zebrafish showed intact main white matter tracts, representing axonal bundles in the forebrain (Fig. 1a) and clearly delineated rhombomere segments in the hindbrain (Fig. 1f). In contrast, the cep41-deficient zebrafish revealed severe axonal tract impairments in both forebrain (Fig. 1b) and hindbrain (Fig. 1g). More than $80 \%$ of cep 41 knockdown zebrafish showed missing or reduced tracts in the anterior commissure (ac), the posterior commissure (pc), and the supra optic tract (sot) of the forebrain and disorganized rhombomere segments in the hindbrain. Remarkably, the 
Table 2 Rare protein altering variants identified in CEP41 in case-control study

\begin{tabular}{lllllllll}
\hline Position & Ref/ Alt & AA change & Case Obs & Control Obs & SIFT & Polyphen & GERP & CADD (Phred) \\
\hline chr7:130038866 & C/G & A330P & 1 & 0 & 0.12 & 0.06 & 2.66 & 7.084 \\
chr7:130040580 & C/T & R242H & 1 & 0 & 0.04 & 0.998 & 6.03 & 34 \\
chr7:130041748 & G/C & P206A & 12 & 1 & 0.14 & 0.997 & 5.54 & 23.3 \\
chr7:130041754 & T/C & M204V & 1 & 0 & 0.15 & 0.64 & 5.54 & 18.04 \\
chr7:130041762 & G/C & S201C & 1 & 0 & 0.03 & 0.997 & 5.54 & 21.9 \\
chr7:130044507 & G/C & A107G & 0 & 1 & 0.39 & 0.116 & 0.835 & 10.79 \\
chr7:130050987 & C/G & A85P & 1 & 0 & 0.28 & 0.773 & 3.18 & 15.08 \\
chr7:130056798 & A/G & M36T & 1 & 0 & 0 & 0.302 & 5.71 & 13.96 \\
\hline
\end{tabular}

SIFT (Sorting Intolerant From Tolerant) predicts impact of amino acid substitutions based on the degree of conservation in sequence alignments derived from closely related sequences. Scores $<0.05$ are considered deleterious. PolyPhen-2 (Polymorphism Phenotyping v2) predicts impact of a variant on the structure and function of a human protein using eight sequence-based and three structure-based predictive features. Scores $>0.95$ are considered probably damaging. GERP (Genomic Evolutionary Rate Profiling) identifies functional constraint of a sequence variant by quantifying substitution deficits in multiple alignments. Substitution deficits represent a natural measure of constraint that reflects the strength of past purifying selection. Higher GERP scores are more deleterious. CADD (Combined Annotation Dependent Depletion) is a framework that integrates multiple annotations into one metric by contrasting variants that survived natural selection with simulated mutations. Higher scores indicate increased deleteriousness

zebrafish expressing CEP41 p.P206A revealed similar axonal defects to those of the cep 41 knockdown zebrafish (Fig. 1c, h), whereas the zebrafish expressing p.A107G variant found in control individuals produced relatively mild axonal defects (Fig. 1d, i). Moreover, the axonal deficits were observed in the zebrafish expressing additional CEP41 variants identified in ASD cases: p.R242H, p. S201C, p.M30T, p.A85P, p.M204V and p.A330P (Supplementary Figure 2).

We further tested the function of ASD-associated CEP41 variants in cep41 knockdown zebrafish in order to demonstrate the specificity of the axonal impairments driven by expression of CEP41 variants. Exogenously expressed WT CEP41 largely restored the axonal deficits of cep41-deficient zebrafish (Supplementary Figure 3b and $3 \mathrm{~m}$ ). The p.A107G variant identified in controls restored the axonal impairments (Supplementary Figure 3d and 3o), whereas p.P206A (Supplementary Figure 3c and $3 n$ ) and six additional variants (Supplementary Figure $3 e-j$ and $3 p-u$ ) identified in ASD cases failed to rescue the defects in both forebrain and hindbrain axonal tracts. Taken together, these results suggest that the CEP41 variants identified in ASD affect axonal tract formation indicating that the role of CEP41 in axonal development may be a mechanism responsible for ASD pathology.

\section{CEP41 is involved in migration of CNC cells in zebrafish}

In ASD, deficits in neuronal migration were proposed as one of the pathophysiological mechanisms ${ }^{39,40}$ and our functional enrichment analysis identified biological processes related to "neuron development" (GO:0048666) in VOI candidate gene analysis. We used a zebrafish model of CNC cell migration as a proxy to evaluate the effects of CEP41 variants on neuronal development. Such a model was previously used to evaluate effects of DISC1, a gene implicated in brain development and multiple brain disorders including autism ${ }^{41,42}$. The depletion of cep41 and expression of p.P206A variant caused delayed migration of CNC cells during 8-12 somites stage in zebrafish brain (Fig. 2). The migrated CNC cells were not observed in the midbrain and the forebrain of cep41-deficient zebrafish at 8 and 10 somite stages, respectively (Fig. $2 \mathrm{~d}-\mathrm{e}, \mathrm{g}-\mathrm{h}$ ). Similarly to what was observed in disc1 morphants ${ }^{42}$, the cep41-defective zebrafish revealed medial expansion, along with hindered lateral migration of $\mathrm{CNC}$ cells at 12 somite stages (Fig. 2f', $\mathrm{i}^{\prime}$ ). The CNC cells successfully migrated in the zebrafish co-injected with cep41 MOs and MO-insensitive zebrafish cep41 mRNA (Fig. 2j-1), thereby indicating a specific role of CEP41 in migration of CNC cells. Taken together, these results suggest that CEP41 acts on neural crest cell migration.

\section{CEP41 variants identified in ASD affect zebrafish social behavior}

To determine whether CEP41 variants affect behaviors that characterize ASD, we examined preference for social interactions of the cep41-ablated zebrafish. First, we examined the behavior of 4-month-old WT zebrafish to evaluate our experimental task. For WT fish, we observed that they preferentially spend time in proximity of the group, which is consistent with preference for social interactions (Supplementary Figure 4b). However, 4month-old cep $41+/-$ fish did not show preference for group proximity (Supplementary Figure 4c) indicating that molecular function of CEP41 is associated with zebrafish social behavior.

Next, we evaluated the effects of ASD variants identified in our case-control study on behaviors of $5-6 \mathrm{dpf}$ 


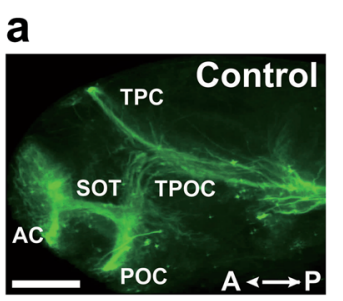

C

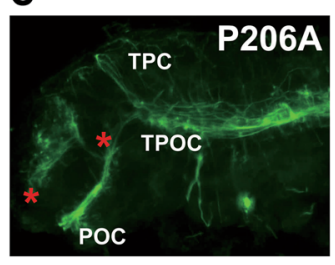

f

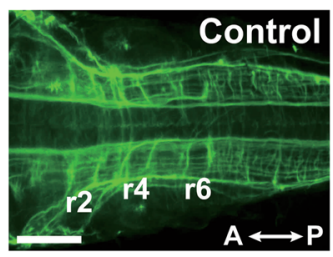

h

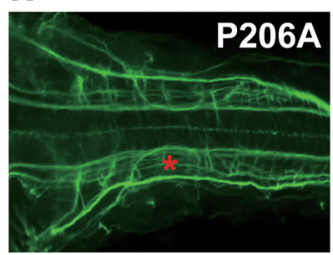

b

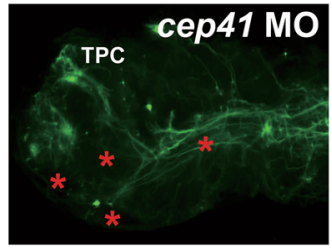

d

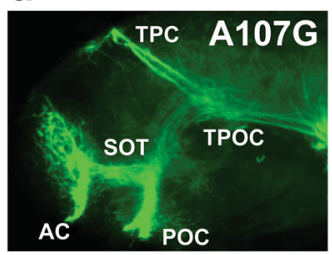

g

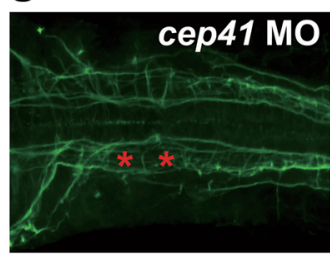

i

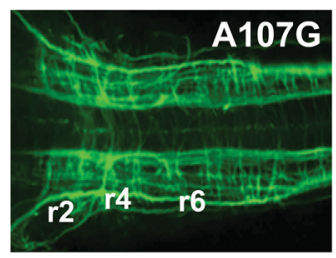

e

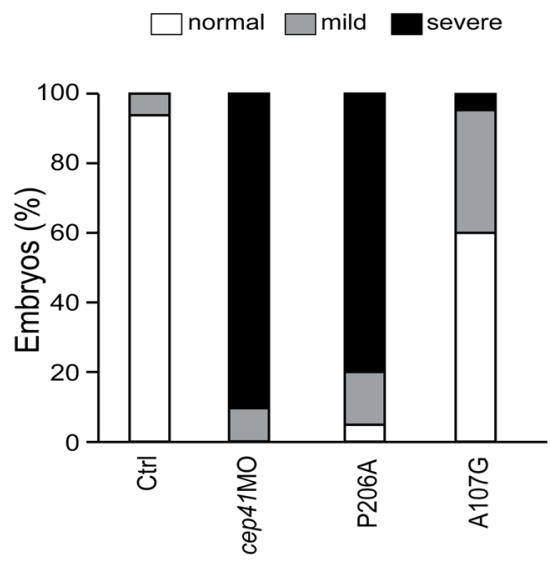

j

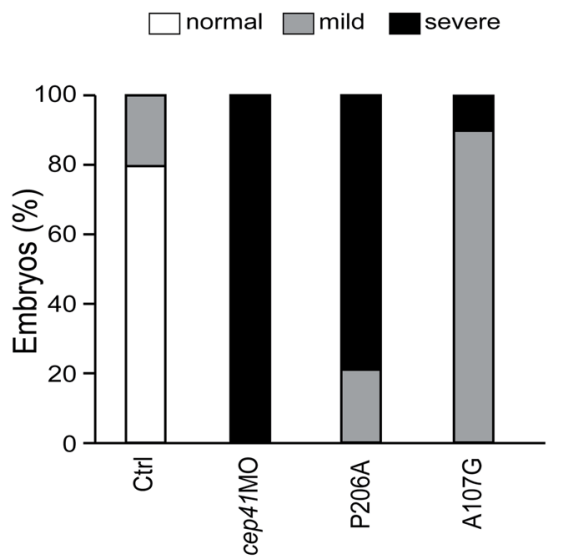

Fig. 1 Expression of human CEP41 variants causes axonal deficits in zebrafish brain. a-d Axon tracts of the forebrain in zebrafish microinjected with cep41 MOs or human CEP41 mRNAs as indicated in each panel. The axons immunostained with anti-acetylated a-Tub antibodies at 35 hpf were imaged by fluorescence microscopy. AC anterior commissures, POC post optic commissure, SOT supra optic tract, TPOC tract of the post optic commissure, TPC tract of the posterior commissure. Asterisks indicate reduced and disorganized axons. Scale bar is $100 \mu \mathrm{m}$. e Quantified data by counting zebrafish embryos with forebrain axonal defects. The severity of defective axons is distinguished as the numbers of malformed axonal tracts. Mild: 1-2 axonal defects. Severe: 3-5 axonal defects. $\mathbf{f}-\mathbf{i}$ Axon tracts of the hindbrain in MOs or mRNA-injected zebrafish. r2, r4 and r6 are rhombomere 2, 4 and 6. Asterisks indicate defective axons and scale bar is $100 \mu \mathrm{m}$. $\mathbf{j}$ Quantified data by counting zebrafish embryos with hindbrain axonal defects. The severity of defected axons is distinguished as the numbers of abnormal axons. Mild: 1-6 axonal defects. Severe: 7-10 axonal defects

zebrafish larvae. We found that control MOs-injected zebrafish exhibit preference for the zone 1 closest to a group (Fig. 3b). For zebrafish with cep 41 knockdown and p.P206A expression, the zone 1 preference was absent, whereas the larvae expressing p.A107G exhibited zone 1 preference in the same way as the control larvae (Fig. $3 \mathrm{c}-\mathrm{e})$. The zebrafish expressing an additional six variants, which were identified in ASD cases, lacked group preference similar to cep41 morphants (Supplementary Figure 5). Further analysis of swimming velocity analysis did not show significant differences between cep41depleted zebrafish larva or adults and controls
(Supplementary Figure 6). Thus, this made it less likely that group preference deficits resulting from cep41 dysfunction are due to dampened swimming mechanics. Finally, the expression of WT CEP41 or the p.A107G variant restored group preference behaviors in the cep41silenced zebrafish (Supplementary Figure $7 \mathrm{~b}$ and d), whereas expression of other variants, including p.P206A, identified in ASD cases failed to do so (Supplementary Figure $7 c$ and $e-j)$. Altogether, these data suggest that CEP41 is important in regulation of social interactions and the variants identified in ASD cases affect the behavioral traits. 

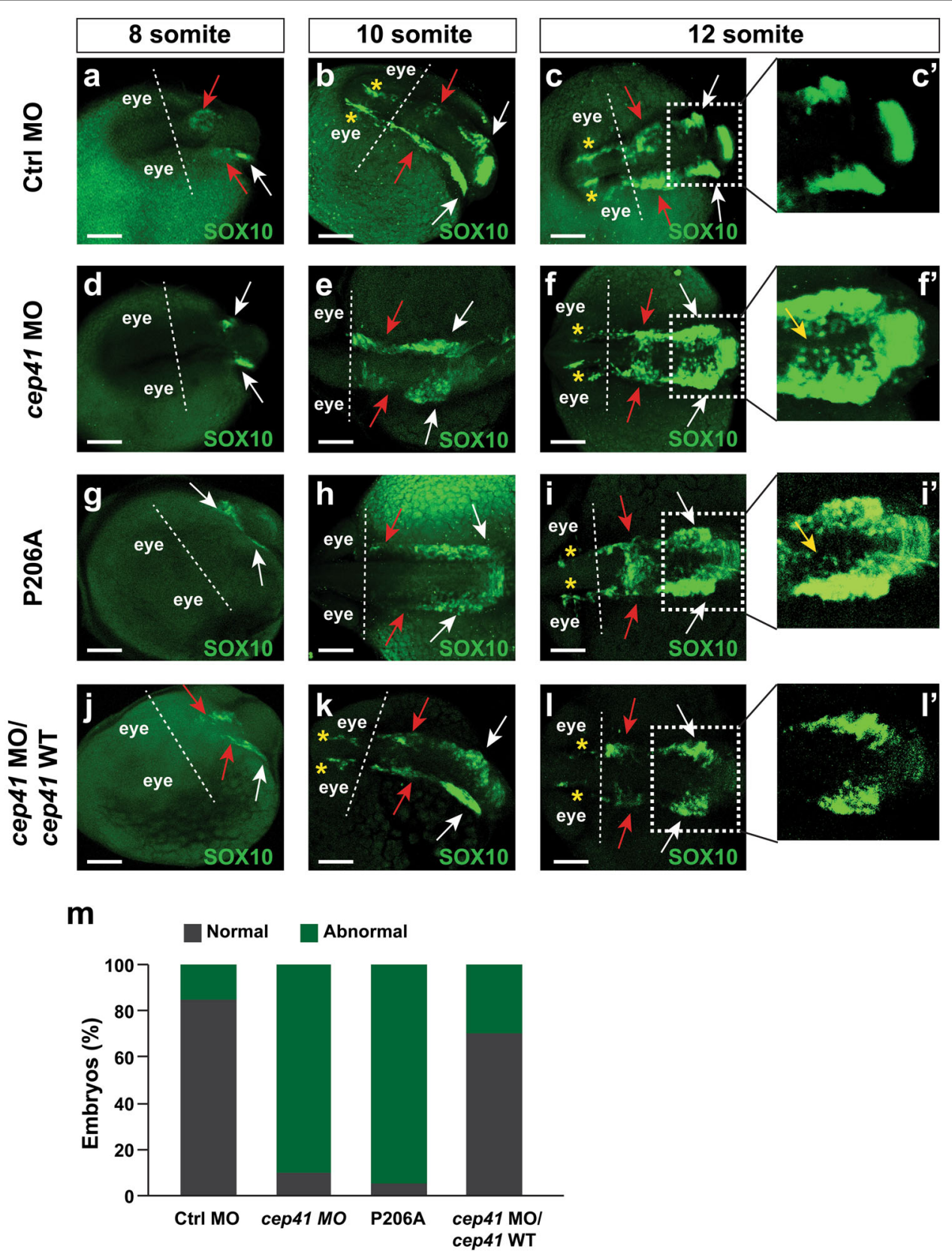

Fig. 2 The depletion of CEP41 affects migration of the cranial neural crest (CNC) cells in zebrafish. Analysis of migration of CNC cells in the control MO (a-c), cep41 MO (d-f), P206A (g-i) and cep41 MO+ zebrafish cep41 mRNA (j-l)-injected zebrafish at 8, 10 and 12 somite stages. Asterisks, red arrows and white arrows indicate migrating CNC cells in the forebrain, midbrain and hindbrain, respectively. The extent of medial expansion of CNC cells (yellow arrows, zoomed in area) was compared in the hindbrain of each zebrafish $\left(\mathbf{c}^{\prime}, \mathbf{f}^{\prime}, \mathbf{i}^{\prime}, \mathbf{I}^{\prime}\right)$. $\mathbf{m}$. All data are representative of at least five independent experiments. The quantified data of zebrafish with defective CNC cell migration are graphically presented (20 embryos/counting).

Abnormal: either delayed migration or medial expansion of CNC cells

\section{Discussion}

Our study has shown that in addition to sporadic autism, NGS-based methods can be successfully applied to the identification of genes involved in familial autism. Our family-based exome sequencing coupled with stringent filtering for private protein altering variants (gene disrupting and missense) has resulted in a limited number of candidate genes. Using a systems biology approach and enrichment analysis, we have shown that candidate genes we identified are enriched for biological processes related to cell projection organization (GO:0030030) and neuron development (GO:0048666). The same biological 
a

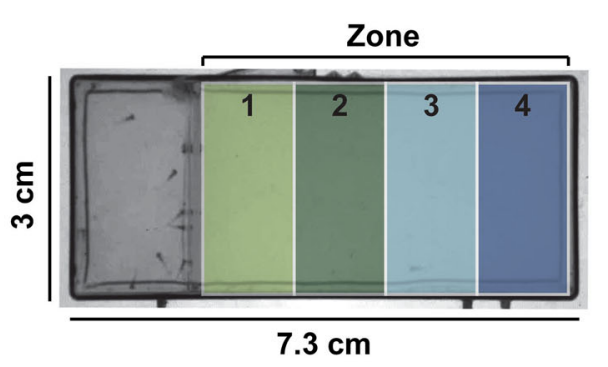

b

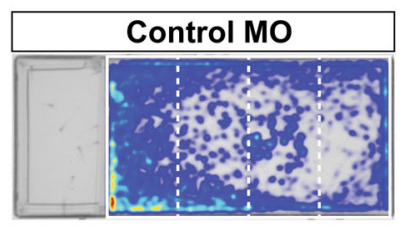

C

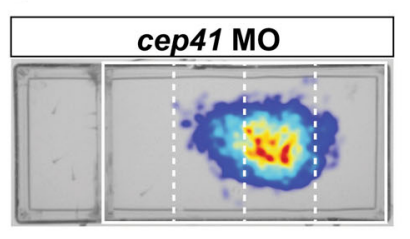

d

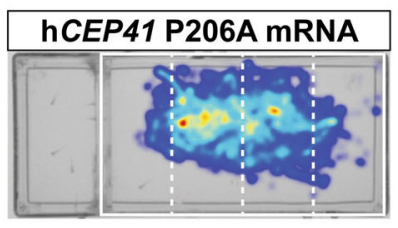

e

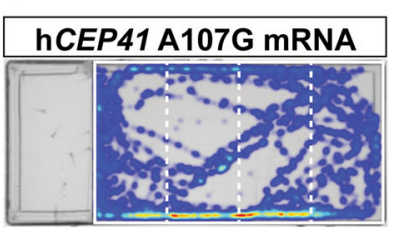

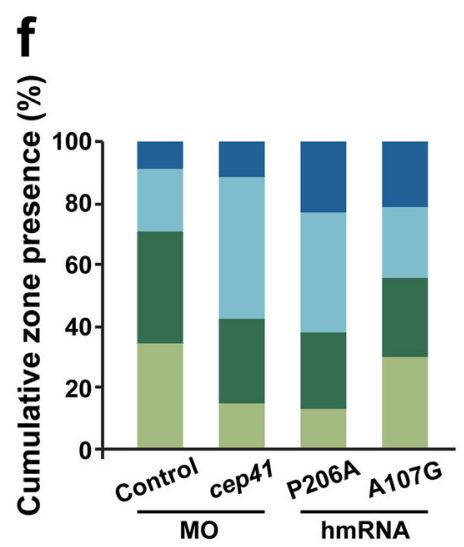

g
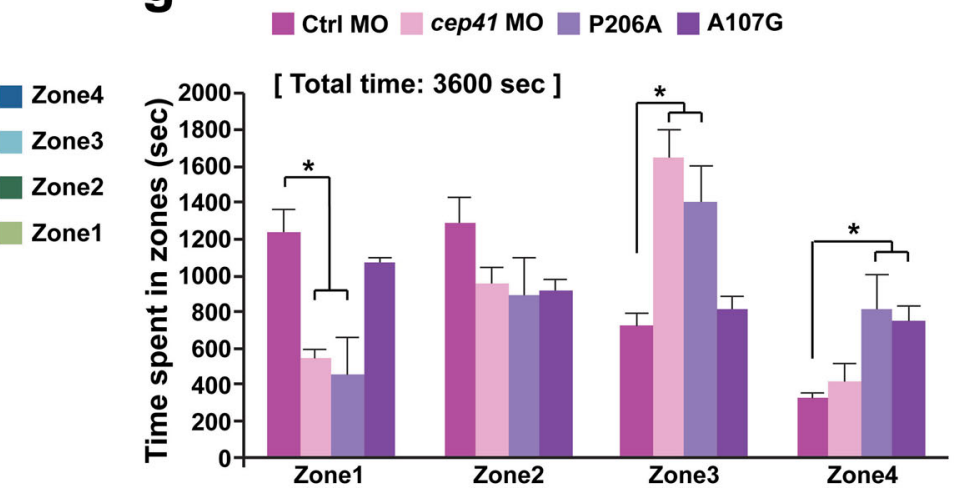

Fig. 3 The deficiency of CEP41 affects social behavior in zebrafish larvae. a A diagram of experimental chamber indicating the zones for the analysis of target location. b-e Heatmap showing the cumulative location of each 5-6 dpf control MO, cep41 MO and human CEP41 P206A and A107G mRNA injected target zebrafish larvae during $1 \mathrm{~h}$ experiment. $\mathbf{f}$ Quantified data presenting the percentage of time spent in each zone during $1 \mathrm{~h}$ experiment. $\mathbf{g}$ The statistical analysis of the time target larvae spent in each zone. The cep41 MO and P206A larvae spend decreased amount of time in zone 1 adjacent to the group. The data are shown as the mean \pm SD; ${ }^{*} p<0.05$ (Student's $t$-test). All data are representative of at least five independent experiments

processes were identified in enrichment analysis of SFARI ASD genes, validating that our candidate gene identification approach was effective. Cell projection organization and neuronal development have been identified as enriched in ASD before. Buxbaum et al. used a systemlevel analysis to evaluate 112 high-risk ASD genes ${ }^{43}$. A high-risk status was assigned to the genes as they are causally implicated in intellectual disability (ID) and the expressed phenotypes include ASD and autistic behaviors ${ }^{44,45}$. Our results expand on findings by Buxbaum et $\mathrm{al}^{43}$, as the candidate genes we identified in enrichment analysis have not been associated with ASD before. In addition, genes in the reported analysis ${ }^{43}$ are all implicated in ID as well. In our study, subjects were ascertained for an ASD phenotype and on average the cognition was above ID cutoff, indicating that the genes we have identified might result in a more narrowly defined ASD phenotype. Furthermore, our enrichment analysis points to genes that are involved in MT cytoskeleton, cell junction and surface and kinase binding, further expanding the understanding of the biological basis of autism.

Our stringent filtering criteria that resulted in a small number of candidate genes in each family allowed us to take a candidate gene approach a step further. Based on Mendelian principles for gene discovery, and the hypothesis that in each family a single gene could be responsible for or be a major risk factor for ASD, we searched the literature to select 13 genes for further targeted sequencing and gene-based analysis in large casecontrol samples. In this way, we identified significant association of rare missense variants in CEP41 with ASD. A prior resequencing study of candidate genes in ASD families positive for $7 \mathrm{q} 32$ linkage signals found rare missense and splice CEP41 variants enriched in ASD cases as compared to controls ${ }^{46}$. In our study, we found statistically significant case-control association of rare CEP41 
variants with ASD, which was supported by family-based TDT association and significant preferential transmission of CEP41 ASD variants to siblings. Furthermore, in a zebrafish model, we showed that these variants have functional effects on brain development and social behavior.

\section{How might CEP41 contribute to autism?}

The CEP41 gene encodes a 41-kDa centrosomal protein that is conserved in vertebrates ${ }^{38,47,48}$. The gene is ubiquitously expressed in human organs including brain ${ }^{49}$. The centrosome is an organelle made of two centrioles that are formed by nine MT triplets embedded in a dense pericentriolar matrix $(\mathrm{PCM})^{50}$. The centrosome serves as the main MT-organizing center of the cell and is a main regulator of cell cycle progression and cell division. In many cell types, following cell cycle division exit, the centrosome converts into a basal body and primary cilium, thus extending the centrosomal role to cell motility and polarity ${ }^{51}$. Centrosomes have been implicated in multiple processes during brain development including neurogenesis and neuronal migration ${ }^{52}$. Proteins that are part of the PCM and interact with MTs have been implicated in a broad spectrum of neurodevelopmental microcephalies (disorders with reduced brain size) and malformations of cortical development such as lissencephaly ${ }^{53-56}$.Therefore, the localization of CEP41 in the MT-based cellular organelles including centrosomes implicates that MT could play a role in the pathogenesis of ASD. Given that MT dynamics is important in the migration of neural crest cells ${ }^{57}$, CEP41 may regulate the dynamic process of MT assembly/disassembly in the centrosome or the axon of neural crest cells during their migration.

In addition to centrosomes, CEP41 involvement in ASD could be mediated through the protein's role in cilia. The dysfunction of cilia is associated with a wide range of human diseases that are known as ciliopathies ${ }^{58}$. Cilia have a function in signal transduction, are ubiquitously expressed, and ciliary dysfunction encompasses most human organ systems with brain, sensory organs, kidneys, skeletal and endocrine/metabolic systems most frequently affected. The phenotypes resulting from ciliary dysfunction have been described as Joubert syndrome (JBTS MIM \#213300), Meckel syndrome (MKS MIM \#249000) and Bardet-Biedel syndrome (BBS MIM \#209900). To date, more than 180 genes have been implicated in 35 established ciliopathies, with numbers continuing to grow. Homozygous CEP41 splice-site mutations have been associated with JBTS, a neurodevelopmental ciliopathy characterized by hypotonia, developmental delays, distinctive MRI cerebellar and brain stem malformation, and a range of physical manifestations that can include ocular, renal, hepatic and endocrine abnormalities ${ }^{38}$.
Furthermore, autism has been described as a part of the JBTS phenotype $\mathrm{e}^{59}$ and genes associated with ciliary dysfunction have been reported in ASD as well (for example: AHI1, CEP290, RPGRIP1L, GUCY2D, RPE65) ${ }^{44}$.

Our study for the first time reported that CEP41 is involved in axon development and $\mathrm{CNC}$ migration. We demonstrated that zebrafish carrying CEP41 variants identified in ASD cases as well as cep 41 mutant/morphants have altered social behavior as manifested by their performance on a test of preference for social interaction. We further showed that these behavioral changes are not likely to be due to swimming abnormalities as we did not observe changes in swimming velocity for cep41-depleted zebrafish. The putative mechanism for how axonal and $\mathrm{CNC}$ migration deficits might cause ASD is through disruption in brain connectivity. Indeed, brain connectivity disruption has been implicated in ASD through neuroimaging and EEG connectivity studies ${ }^{60,61}$.

\section{Limitations and future directions}

Although we identified the association of CEP41 with ASD, our study design did not provide evidence for association of a specific candidate gene with autism in 25 out of 26 families. This is not surprising as ASD is highly heterogeneous and any repository, even if very large, may not be sufficiently enriched for a specific genetic subtype. We identified a total of 145 VOIs in 139 genes in 20 of 26 families and we have shown that the identified genes are enriched for biological processes such as neuronal development and cell projection organization that are implicated in ASD. Given the need for large number of cases and controls, it was only feasible to sequence 13 candidate genes for association analysis. Our enrichment analysis further supports that some of the 126 genes we have not evaluated in case-control study might be associated with ASD as well and this could be tested in future studies.

The involvement of CEP41, first in the ciliopathy JBTS and now with our study in ASD, points toward gene dosage and variant-specific effect as factors that determine the phenotype. In JBTS, the implicated variants are homozygous splice site-disrupting variants. In ASD, we have found that implicated variants are heterozygous and missense. Instances of different types of variants and gene dosage levels leading to distinctive phenotypes have been described in ciliopathies. For example, gene disrupting nonsense mutations in $C C 2 D 2 A$ gene cause Meckel-Gruber syndrome (MKS) with severe phenotype ${ }^{62}$, whereas missense mutations in the same gene cause JBTS where the phenotype is less severe ${ }^{63}$. In BBS ciliopathy, gene dosage is an important modulator of phenotype. Retinal disease is one of the cardinal features in recessive BBS with homozygous mutations. Although heterozygous carriers do not have overt visual disability, 
electroretinography reveals decreased sensitivity of photoreceptor response in all parents of children with $\mathrm{BBS}^{64}$, indicating that gene dosage quantitatively affects the phenotype. For CEP41, the heterozygous state with one WT allele and one allele with a missense mutation might not perturb the biological system to the extent that it would cause severe neurodevelopment consequences such as in JBTS where both copies of the gene have a protein-disrupting mutation, but it could still lead to lower level of perturbation that is phenotypically expressed as ASD. Furthermore, as JBTS ciliopathies, including the CEP41 subtype, are characterized by distinctive cerebellar malformation signature (i.e. molar tooth sign), the individuals with ASD and CEP41 heterozygous mutations should be investigated with neuroimaging to evaluate for structural CNS abnormalities.

Our enrichment analysis also implies that other genetic mechanisms, besides single gene causality that we evaluated by case-control study, might be contributory for autism. The ciliopathies clearly demonstrate the involvement of a digenic mechanism $^{58,65}$. The proteins coded by genes which are implicated in ciliopathies form complexes of physically closely interacting proteins ${ }^{66}$. Heterozygous defects in two closely interacting genes can cause biological disruption in a similar way as compound heterozygosity in a single gene. Indeed, a digenic mechanism has been implicated in CEP41 ciliopathies; heterozygous missense $C E P 41$ variants coupled with heterozygous $K I F 7$ or $C C 2 D 2 A$ variants were reported in individuals with ciliopathy ${ }^{38}$. A focus on ciliopathy genes and biologically defined molecular targets narrows the number of genes to be evaluated, thus significantly reducing the required sample size and burden of multiple testing for digenic mechanisms. In the future, involvement of a digenic mechanism for ciliopathy genes in ASD could be tested using currently publically available resources. For example, ASD genetics resources such as MSSNG (https://www.mss. ng/) and the Simons Simplex Collection (https://www.sfari. org/resource/simons-simplex-collection/) have a large number of exome and whole-genome sequenced autism cases and controls. For the approximately 180 genes associated with ciliopathies, one could evaluate whether digenic VOIs are seen more frequently in cases as compared to controls; and in such a way explore statistical evidence for a digenic mechanism in ASD.

\section{Acknowledgements}

We acknowledge the clinicians, organizations and families that contributed to data and samples used in this study (https://www.nimhgenetics.org/ acknowledgements.php). DNA samples and phenotype information were obtained from NIMH Center for Collaborative Genomic Research on Mental Disorders. Research reported in this publication was supported by funding from the National Institute of Mental Health under award numbers R01 MH092367 from the National Institutes of Health, and from the Korean Government (MSIP) (NRF-2016R1CIB2008930 and 2017R1E1A2A01076144). The zebrafish study was reviewed and approved by the Institutional Animal Care and Use Committee (IACUC) of Samsung Biomedical Research Institute (SBRI).

\section{Author details}

'Department of Psychiatry and Behavioral Sciences, University of Washington, Seattle, WA, USA. 'Department of Health Sciences and Technology, SAIHST, Sungkyunkwan University, Seoul, Korea. ${ }^{3}$ Department of Genome Sciences, University of Washington, Seattle, WA, USA. ${ }^{4}$ Department of Medicine, Division of Medical Genetics, University of Washington, Seattle, WA, USA. ${ }^{5}$ Division of Medical Science Research, Samsung Medical Center, Seoul, Korea

\section{Conflict of interest}

The authors declare no conflict of interest.

\section{Publisher's note}

Springer Nature remains neutral with regard to jurisdictional claims in published maps and institutional affiliations.

Supplementary Information accompanies this paper at (https://doi.org/ 10.1038/s41398-018-0343-z).

Received: 9 August 2018 Accepted: 13 November 2018

Published online: 15 January 2019

\section{References}

1. Association AP. Diagnostic and Statistical Manual of Mental Disorders: Dsm-5. Washington, DC (American Psychiatric Publication Incorporated, 2013).

2. Sandin, S. et al. The familial risk of autism. JAMA 311, 1770-1777 (2014).

3. Ronald, A. \& Hoekstra, R. A. Autism spectrum disorders and autistic traits: a decade of new twin studies. Am. J. Med. Genet. B Neuropsychiatr. Genet. 156b, 255-274 (2011).

4. Turner, T. N. et al. Genome sequencing of autism-affected families reveals disruption of putative noncoding regulatory DNA. Am. J. Hum. Genet. $\mathbf{9 8}$ 58-74 (2016)

5. O'Roak, B. J. et al. Multiplex targeted sequencing identifies recurrently mutated genes in autism spectrum disorders. Science 338, 1619-1622 (2012).

6. O'Roak, B. J. et al. Sporadic autism exomes reveal a highly interconnected protein network of de novo mutations. Nature 485, 246-250 (2012).

7. lossifov, I. et al. The contribution of de novo coding mutations to autism spectrum disorder. Nature 515, 216-221 (2014).

8. Krumm, N., O'Roak, B. J., Shendure, J. \& Eichler, E. E. A de novo convergence of autism genetics and molecular neuroscience. Trends Neurosci. 37, 95-105 (2014).

9. Yuen, R. K. et al. Whole-genome sequencing of quartet families with autism spectrum disorder. Nat. Med. 21, 185-191 (2015).

10. De Rubeis, S. et al. Synaptic, transcriptional and chromatin genes disrupted in autism. Nature 515, 209-215 (2014).

11. Li, J. et al. Targeted sequencing and functional analysis reveal brain-size-related genes and their networks in autism spectrum disorders. Mol. Psychiatry 22, 1282-1290 (2017).

12. An, J. Y. et al. Towards a molecular characterization of autism spectrum disorders: an exome sequencing and systems approach. Transl. Psychiatry 4, e394 (2014).

13. Pinto, D. et al. Functional impact of global rare copy number variation in autism spectrum disorders. Nature 466, 368-372 (2010).

14. Devlin, B. \& Scherer, S. W. Genetic architecture in autism spectrum disorder. Curr. Opin. Genet. Dev. 22, 229-237 (2012).

15. Chahrour, M. H. et al. Whole-exome sequencing and homozygosity analysis implicate depolarization-regulated neuronal genes in autism. PLoS Genet. 8, e1002635 (2012).

16. Al-Mubarak, B. et al. Whole exome sequencing reveals inherited and de novo variants in autism spectrum disorder: a trio study from Saudi families. Sci. Rep. 7, 5679 (2017).

17. $\mathrm{Yu}, \mathrm{T}$. et al. Using whole exome sequencing to identify inherited causes of autism. Neuron 77, 259-273 (2013).

18. Shi, L. et al. Whole-genome sequencing in an autism multiplex family. Mol. Autism 4, 8 (2013).

19. Egawa, J. et al. Novel rare missense variations and risk of autism spectrum disorder: whole-exome sequencing in two families with affected siblings and 
a two-stage follow-up study in a Japanese population. PLoS ONE 10, e0119413 (2015).

20. Inoue, E. et al. Resequencing and association analysis of CLN8 with autism spectrum disorder in a Japanese population. PLOS ONE 10, e0144624 (2015).

21. Toma, C. et al. Exome sequencing in multiplex autism families suggests a major role for heterozygous truncating mutations. Mol. Psychiatry 19, 784-790 (2014).

22. Cukier, H. N. et al. Exome sequencing of extended families with autism reveals genes shared across neurodevelopmental and neuropsychiatric disorders. Mol. Autism 5, 1 (2014)

23. Chapman, N. H. et al. Whole exome sequencing in extended families with autism spectrum disorder implicates four candidate genes. Hum. Genet. 134, 1055-1068 (2015).

24. Yuen, R. K. et al. Whole genome sequencing resource identifies 18 new candidate genes for autism spectrum disorder. Nat. Neurosci. 20, 602-611 (2017).

25. Sham, P. C. \& Purcell, S. M. Statistical power and significance testing in largescale genetic studies. Nat. Rev. Genet. 15, 335-346 (2014).

26. Rubinstein, $M$. et al. Association of rare missense variants in the second intracellular loop of NaV1.7 sodium channels with familial autism. Mol. Psychiatry 23, 231-239 (2016).

27. O'Roak, B. J. et al. Exome sequencing in sporadic autism spectrum disorders identifies severe de novo mutations. Nat. Genet. 43, 585-589 (2011).

28. Li, H. \& Durbin, R. Fast and accurate short read alignment with BurrowsWheeler transform. Bioinformatics 25, 1754-1760 (2009).

29. McKenna, A. et al. The Genome Analysis Toolkit: a MapReduce framework for analyzing next-generation DNA sequencing data. Genome Res. 20, 1297-1303 (2010).

30. Wang, K., Li, M. \& Hakonarson, H. ANNOVAR: functional annotation of genetic variants from high-throughput sequencing data. Nucleic Acids Res. 38, e164 (2010).

31. Boyle, E. A., O'Roak, B. J., Martin, B. K, Kumar, A. \& Shendure, J. MIPgen: optimized modeling and design of molecular inversion probes for targeted resequencing. Bioinformatics 30, 2670-2672 (2014).

32. Rehker, J. et al. Caspase-8, association with Alzheimer's Disease and functional analysis of rare variants. PLOS ONE 12, e0185777 (2017).

33. He, Z. et al. Rare-variant extensions of the transmission disequilibrium test: application to autism exome sequence data. Am. J. Hum. Genet. 94, 33-46 (2014).

34. Wang, J., Vasaikar, S., Shi, Z., Greer, M. \& Zhang, B. WebGestalt 2017: a more comprehensive, powerful, flexible and interactive gene set enrichment analysis toolkit. Nucleic Acids Res. 4, 36 (2017).

35. Abrahams, B. S. et al. SFARI Gene 2.0: a community-driven knowledgebase for the autism spectrum disorders (ASDs). Mol Autism 4, 36 (2013).

36. Kas, M. J. et al. Assessing behavioural and cognitive domains of autism spectrum disorders in rodents: current status and future perspectives. Psychopharmacology (Berl) 231, 1125-1146 (2014).

37. Stewart, A. M., Nguyen, M., Wong, K., Poudel, M. K. \& Kalueff, A. V. Developing zebrafish models of autism spectrum disorder (ASD). Prog. Neuropsychopharmacol. Biol. Psychiatry 50, 27-36 (2014).

38. Lee, J. E. et al. CEP41 is mutated in Joubert syndrome and is required for tubulin glutamylation at the cilium. Nat. Genet. 44, 193-199 (2012).

39. Reiner, O., Karzbrun, E., Kshirsagar A. \& Kaibuchi, K. Regulation of neuronal migration, an emerging topic in autism spectrum disorders. J. Neurochem. 136, 440-456 (2016).

40. Wegiel, J. et al. The neuropathology of autism: defects of neurogenesis and neuronal migration, and dysplastic changes.

41. Hikida, T., Gamo, N. J. \& Sawa, A. DISC1 as a therapeutic target for mental illnesses.
42. Drerup, C. M. et al. Disc1 regulates foxd3 and sox10 expression, affecting neural crest migration and differentiation. Development 136, 2623-2632 (2009).

43. Buxbaum, J. D.et al. Optimizing the phenotyping of rodent ASD models: enrichment analysis of mouse and human neurobiological phenotypes associated with high-risk autism genes identifies morphological, electrophysiological, neurological, and behavioral features. Mol. Autism 3, 1 (2012).

44. Betancur, C. Etiological heterogeneity in autism spectrum disorders: more than 100 genetic and genomic disorders and still counting. Brain Res. 1380, 42-77 (2011).

45. Neale, B. M. et al. Patterns and rates of exonic de novo mutations in autism spectrum disorders. Nature 485, 242-245 (2012).

46. Korvatska, $\mathrm{O}$. et al. Mutations in the TSGA14 gene in families with autism spectrum disorders. Am. J. Med. Genet. B Neuropsychiatr. Genet. 156b 303-311 (2011)

47. Andersen, J. S. et al. Proteomic characterization of the human centrosome by protein correlation profiling. Nature 426, 570-574 (2003).

48. Gache, V. et al. Xenopus meiotic microtubule-associated interactome. PLoS ONE 5, e9248 (2010).

49. Yamada, T. et al. The gene TSGA14, adjacent to the imprinted gene MEST escapes genomic imprinting. Gene $\mathbf{2 8 8}$, 57-63 (2002).

50. Meraldi, P. \& Nigg, E. A. The centrosome cycle. FEBS Lett. 521, 9-13 (2002).

51. Bettencourt-Dias, M. \& Glover, D. M. Centrosome biogenesis and function: centrosomics brings new understanding. Nat. Rev. Mol. Cell. Biol. 8, 451-463 (2007).

52. Chavali, P. L., Putz, M. \& Gergely, F. Small organelle, big responsibility: the role of centrosomes in development and disease. Philos. Trans. R. Soc. Lond. B. Biol. Sci. 369, 20130468 (2014).

53. Mochida, G. H. Genetics and biology of microcephaly and lissencephaly. Semin. Pediatr. Neurol. 16, 120-126 (2009).

54. Kuijpers, M. \& Hoogenraad, C. C. Centrosomes, microtubules and neuronal development. Mol. Cell. Neurosci. 48, 349-358 (2011).

55. Barkovich, A. J. et al. A developmental and genetic classification for malformations of cortical development: update. Brain 135, 1348-1369 (2012).

56. Bonini, S. A., Mastinu, A., Ferrari-Toninelli, G. \& Memo, M. Potential role of microtubule stabilizing agents in neurodevelopmental disorders. Int. J. Mol. Sci. 18, 1627 (2017).

57. Moor, R. et al. Par3 controls neural crest migration by promoting microtubule catastrophe during contact inhibition of locomotion. Development 140, 4763-4775 (2013)

58. Reiter, J. F. \& Leroux, M. R. Genes and molecular pathways underpinning ciliopathies.

59. Miles, J. H. Autism spectrum disorders-a genetics review.

60. O'Reilly, C., Lewis, J. D. \& Elsabbagh, M. Is functional brain connectivity atypical in autism? A systematic review of EEG and MEG studies.

61. Mohammad-Rezazadeh, I., Frohlich, J., Loo, S. K. \& Jeste, S. S. Brain connectivity in autism spectrum disorder. Curr. Opin. Neurol. 29, 137-147 (2016).

62. Tallila, J., Jakkula, E., Peltonen, L., Salonen, R. \& Kestilä, M. Identification of CC2D2A as a Meckel Syndrome gene adds an important piece to the ciliopathy puzzle. Am. J. Hum. Genet. 82, 1361-1367 (2008).

63. Gorden, N. T. et al. CC2D2A is mutated in Joubert Syndrome and interacts with the ciliopathy-associated basal body protein CEP290. Am. J. Hum. Genet 83, 559-571 (2008).

64. Cox, G. F., Hansen, R. M., Quinn, N. \& Fulton, A. B. Retinal function in carriers of Bardet-Biedl syndrome. Arch. Ophthalmol. 121, 804-810 (2003).

65. Schäffer, A. A. Digenic inheritance in medical genetics. J. Med. Genet. 50, 641-652 (2013).

66. Yee, L. E. et al. Conserved genetic interactions between ciliopathy complexes cooperatively support ciliogenesis and ciliary signaling. PLOS Genet. 11, e1005627 (2015). 